\title{
Emerging Single-cell Approaches to Understand HIV in the Central Nervous System
}

\author{
Michael J. Corley ${ }^{1} \cdot$ Shelli F. Farhadian ${ }^{2,3}$ (1) \\ Accepted: 5 October 2021 / Published online: 25 November 2021 \\ (c) The Author(s), under exclusive licence to Springer Science+Business Media, LLC, part of Springer Nature 2021
}

\begin{abstract}
Purpose of Review This review highlights emerging single-cell sequencing methods relevant to translational studies of HIV in the central nervous system (CNS), summarizes limited single-cell studies of HIV in the CNS, and discusses opportunities for future HIV translational CNS studies.

Recent Findings Innovative methods utilizing single-cell technologies have advanced the study of genomes, proteomes, transcriptomes, and epigenomes at an enhanced resolution and depth. Single-cell analyses of central nervous system tissue, including autopsy brain and CSF cells, may shed light on CNS perturbations in people living with HIV. New strategies can distinguish distinct molecular identifies of rare infected cells at single-cell level, suggesting an opportunity to uncloak the molecular identity of hidden HIV in the CNS reservoir.

Summary Adoption of multimodal "omics" analyses to translational HIV studies and tissue compartments beyond blood will be critical to advancing our understanding of viral establishment, persistence, and eradication.
\end{abstract}

Keywords HIV $\cdot$ CNS $\cdot$ Single cell $\cdot$ Cerebrospinal fluid

\section{Introduction}

Human immunodeficiency virus (HIV) remains an enigmatic human retrovirus without a cure despite advancements in our understanding of disease pathogenesis and its treatment [1]. While advancements in antiretroviral therapy (ART) have led to tremendous improvements in the lives of people living with HIV (PLWH), the virus persists in cellular reservoirs throughout the body and viral rebound ensues predictably following antiretroviral treatment interruption [2]. Only two people, the "Berlin" [3] and "London" patients [4], have

Michael J. Corley

mjc4002@med.cornell.edu

$\bowtie$ Shelli F. Farhadian shelli.farhadian@yale.edu

1 Deparment of Medicine, Division of Infectious Diseases, Weill Cornell Medicine, 413 East 69th Street, Belfer Research Building, 5th floor, BB-514, New York, NY 10021, USA

2 Department of Medicine, Section of Infectious Diseases, Yale School of Medicine, New Haven, CT 06520, USA

3 Department of Neurology, Yale School of Medicine, 135 College St, Suite 378, New Haven, CT 06520, USA been cured of HIV following unique and arduous treatment regimens. All other HIV cure and remission strategies have had limited success in PLWH, indicating an ongoing need to better understand both the diversity of PLWH and the complexity of HIV at all stages of infection, and in tissue compartments throughout the body [5, 6].

During acute infection, in most individuals, HIV enters the immune privileged central nervous system, causing CSF viremia, and establishing local infection in the brain [7, 8]. Autopsy studies of PLWH who died while on ART find HIV DNA in the brain of most subjects $[9,10]$, and studies of HIV RNA sequences from CSF specimens suggest that HIV replicates in the CNS in both T cells and macrophages, forming a compartmentalized infection with genetic features distinct from paired sequences found in the plasma [11]. Viral burden within the CNS is associated with neuroinflammation, neuronal injury, and clinical neurological deficits [12-14]. Thus, the CNS is an important viral reservoir site of HIV that contributes to HIV morbidity through chronic immune activation and potentially through ongoing viral replication in the CNS. Yet, compared to the level of investigation of peripheral blood and the CD4 + T lymphocyte HIV reservoir in studies of PLWH, the CNS compartment remains understudied. Routine access to brain tissue in 
PLWH is challenging; hence, lumbar puncture and the collection of CSF has been utilized as a window into studying the CNS compartment in PLWH [15]. CSF studies are safe in PLWH and feasible even in international research settings and during the earliest stages of acute HIV infection [16•].

Technological advances in single-cell technologies based on next generation sequencing now allow for the study of single human cells at an unprecedented level of resolution. In particular, recent single-cell technologies permit the high-throughput study of individual cellular genomes, proteomes, transcriptomes, epigenomes, metabolomes, and multiomes [17] in clinical samples, and, through cellular infection assays, allow for detailed study of host-pathogen interactions [18]. These methods have been rapidly adopted and utilized to understand emerging infectious diseases with neuropathology such as COVID-19 and Zika [19-21]. The application of single cell sequencing technologies in the HIV field has emerged as an essential tool for studying perturbations to host immune cells during systemic HIV infection,

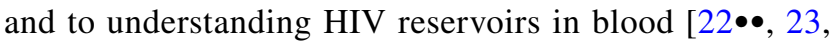
$24 \bullet \bullet, 25 \bullet \bullet, 26]$. However, the application of single-cell omic technologies to human HIV studies of the CNS remain limited. In this review, we overview new single-cell omics technologies extending beyond the transcriptome, summarize translational HIV CNS studies using single-cell profiling, and highlight the potential application of single-cell omics studies to neuro-immune responses during HIV infection and CNS viral reservoirs.

\section{Single-cell Sequencing Approaches to Characterize Host Immune Responses in the CNS During HIV Infection}

\section{Modern Single-cell RNA Transcriptome Sequencing}

The most widely utilized single-cell sequencing technology has been single-cell mRNA sequencing that provides a quantitative assessment of the transcriptome profile of individual cells [27]. For a review of the history and advances in singlecell sequencing see the following reviews [28, 29]. Briefly, high-throughput single-cell RNA sequencing technologies were developed that rely on droplet-based methods [30•, $31]$ to increase the scale and throughput of cells that are profiled. These approaches scaled low-throughput single-cell sequencing approaches that utilized fluorescence-activated cell sorting of rare cells into 96 or 384 well plates or capturing of rare germ cells for single-cell transcriptiome profiling [27]. By far, the leading single-cell laboratory instrument has been the commercial platform provided by $10 \times$ Genomics Chromium that is based on microfluidics with molecular barcoding [32]. Innovative "super-loading" approaches that utilize multiplexing of cells by either oligo-tagged antibodies directed against ubiquitously expressed immune surface markers (Cell Hashing [33]) or lipid- and cholesterol-modified oligonucleotides (MULTI-Seq [34•]) have reduced the cost of profiling per cell and enhanced the throughput of the $10 \times$ Genomics instrument by five to ten-fold. The "super loading" innovation for single-cell RNA sequencing is a key development for the HIV field, which aims to profile extremely rare cells containing HIV RNA to identify cellular reservoirs that are transcription competent [35].

Modifications to validated antibodies typically utilized in the gold standard assay of flow cytometry with unique oligonucleotide conjugates developed in immune-polymerasse chain reaction (immune-PCR) [36] have permitted the detection of surface proteins by converting amplifiable DNA sequence into an inferred protein signal. When utilized in conjunction with single-cell RNA-seq microfluidics-based or nano-well cartridge-based methods, these approaches (CITE-Seq [37•], REAP-Seq [38], AbSeq [39]) have shown that both RNA and protein can be simultaneously detected in single cells. Innovative approaches have modified the single-cell protein detection methods to expand beyond surface protein labeling and have shown that intracellular protein detection is possible at single-cell level in a method termed intranuclear cellular indexing of transcirptomes and epitopes (inCITE-seq) [40]. Application of these single-cell based sequencing approaches with modifying a p24-based method developed on flow cytometry to identify translational-competent viral reservoirs [41] has the potential to advance the study of rare, HIV-infected cells in the CNS compartment.

\section{Single-cell Studies of Cerebrospinal Fluid Immune Cells in Neuroinflammatory Conditions}

Cerebrospinal fluid (CSF) immune cells provide a window into infiltrating brain parenchymal immune cells. Hence, studies have utilized scRNA-seq of CSF to interrogate CNS immune cells during neuroinflammatory conditions,

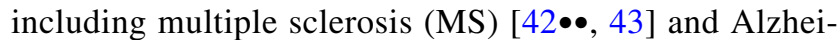

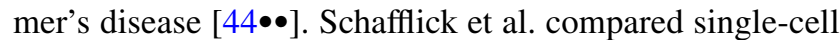
transcriptomic profiles of matched peripheral blood and CSF samples from patients with MS, and found that, compared to controls, individuals with MS have an expansion of cytotoxic CD4 + T cells in the CSF [45]. Recent research has also utilized single-cell RNA-seq to characeterize CSF cells in individuals with acute COVID-19. Heming et al profiled eight individuals with COVID-19 and neurological symptoms and six being characterized as mild to moderate COVID-19 with mild neurological complications[19]. As a comparison group, the authors utilized single-cell RNASeq CSF datasets from individuals with idiopathic intracranial hypertension, MS, and viral encepthalitis. Comparative analyses revealed that COVID-19 was associated with a significant expansion of a monocyte cell cluster with an 
antigen-presenting phenotype, and exhausted T cells. Song and Bartley et al. used single-cell RNA-Seq to compare CSF and blood in COVID-19 patients and controls, and and reported increased signatures of $\mathrm{T}$ cell activation and interferon pathways in the CSF of COVID-19 patients [20]. These studies demonstrate the ability to characterize CNS immune responses during infectious disease through CSFbased single-cell RNA-Seq analyses.

\section{Single-cell Transcriptome Studies of CSF Immune Cells of Individuals Living with HIV on ART}

We previously utilized single-cell RNA-Seq to characterize the immune cell landscape of paired fresh CSF and peripheral blood in PLWH on ART [46•]. Based on transcriptional features, we identified that $\mathrm{T}$ lymphocytes were the majority cell type in CSF, followed by myeloid cells. Moreover, a subanalysis of myeloid cells in the CSF revealed that a unique myeloid cell subset characterized by elevated gene expression of genes enriched in neurodegenerative diseaseassociated microglia (APOE, AXL, and TREM2) was present in CSF, but not the blood, of people living with HIV on ART, consistent with later studies that also identify myeloid lineage cells that are unique to the CSF [43, 45, 47]. This study demonstrated that single-cell transcriptional studies of CSF in people living with HIV are feasible, and that a subset of myeloid cells might contribute to neuropathogenesis during HIV infection.

\section{Single B cell and T cell Receptor Sequencing in CNS}

Sequencing of T cell receptor (TCR) and B cell receptor (BCR) genes at the single-cell level can identify expanded clones within tissue compartments that associate with disease state or the presence of viral antigen. Multimodal single TCR and transcriptome sequencing of CSF T cells during COVID-19 [19] and Alzheimer's disease (AD) [44••], demonstrated clonal expansion of T cells within the CNS compartment during these conditions. The population of clonally expanded CD8 T cells in CSF associated with $\mathrm{AD}$ appeared to arise from viral antigenspecific clonal expansion, expressed pro-inflammatory genes, and was not observed in healthy individuals, suggesting a link between antigen-specific clonal expansion of $\mathrm{T}$ cells in the CNS and neurodegeneration. In HIV infection, in peripheral blood, TCR sequencing combined with methods to isolate single HIV-infected CD4 T cells was used to characterize clonal expansion of the HIV reservoir in blood [22••]. It is not known whether CD4 T cell reservoirs in the CNS are likewise clonally expanded in that compartment during chronic HIV infection. Single TCR sequencing can also help elucidate dynamics of the anti-viral CD8 T cell response in the CNS. Prior studies using bulk TCR sequencing suggest that antigen-specific CD8 $\mathrm{T}$ cells clonally expand in the CNS during acute infection [48].
Longitudinal and paired single-cell TCR sequencing from blood and CSF may help address whether these cells persist and continue to expand in response to local antigen stimulation in the CNS over the course of chronic infection.

Identification of antigen-specific TCR sequences at scale remains challenging. Hence, future methods development will scale the ability to label antigen-specific $\mathrm{T}$ cells with multimeric MHC peptide complexes that bind to TCRs with high affinity [49], enabling the identification of multiple antigens or epitopes paired to full-length receptor sequences.

Single BCR sequencing of the CSF may likewise reveal features of the CNS immune response to HIV infection [20]. To identify HIV antigen-specific B cells paired with heavy and light chain B cell receptor sequences at single-cell level, an approach termed LIBRA-Seq has been utilized that relies on conjugating oligonucleotide barcodes to recombinant HIV proteins, which are then used to sort tagged HIV-specific B cells [50•]. LIBRA-Seq was utilized to identify HIV antigenspecific B cells in peripheral blood from two people living with HIV. The application of this approach to CSF would enhance HIV-specific antibody discovery in the CNS.

\section{Single-cell Epigenomics}

HIV infection alters the human epigenetic landscape. Singlecell epigenetic methods, including scATAC-seq, have been developed to study DNA methylation, chromatin accessibility, chromatin interactions, and histone modifications [51]. Moreover, a method termed Cleavage Under Targets and tagmentation (CUT\&Tag) targets a specific protein of interest in situ and permits single-cell genome-wide profiling of chromatin modifications [52•]. When CUT\&TAG is combined with the $10 \times$ Genomics scATAC-Seq protocol to partition single cells, it enables the mapping of histone modifications and transcriptional factors in single cells. These approaches build upon prior work that has characterized the chromatin landscape of bulk HIV-infected cell populations, and shed light on molecular features that allow HIV to integrate into particular sites of the host genome [53]. The capability to profile epigenomes of single cells in the CNS compartment during HIV infection will provide key insights into infection-induced epigenetic changes, the impact on neuropathogenesis of HIV, and have implications for improving our understanding of HIV CNS reservoirs.

\section{Single-cell Approaches to Understanding CNS Viral Reservoirs}

\section{Single-cell RNA and DNA Sequencing to Characterize Proviral Integration}

While prior studies have provided a variety of methods, estimates, and characterizations of the frequency of 
peripheral blood CD4 T cells containing HIV RNA and latent HIV DNA [54], the proportion of CNS immune cells harboring HIV remains unclear. Unique cohorts of PLWH who have generously donated their bodies for postmortem study provide an opportunity to better characterize the HIV brain reservoir using single-cell methods [55]. Using tissue from the National NeuroAIDS Tissue Consortium, Plaza-Jennings et al assessed brain tissue from PLWH with and without HIV encephalitis using single nuclei RNA sequencing of isolated postmortem brain cell nuclei, a method that allows for single-cell transcriptomic study of fresh frozen postmortem brain tissues. By assessing single-cell transcriptomes for both host and viral genes, they found that $\mathrm{T}$ lymphocytes and microglial cells are the two cell types that harbor HIV in autopsy brain, and begin to characterize integration sites of HIV in brain tissue [56].

Single-cell DNA sequencing approaches further allow for characterization of HIV integration sites, but, to date, have focused exclusively on blood. Sun and collegues recently developed Simultaneous Integration site and Provirus sequencing, SIP-seq [57], which uses whole genome amplification in microfluidic droplets to capture and sequence full-length proviral HIV genomes with their associated integration sites. An approach to simultaneously analyze HIV proviral sequences and integration sites termed STIP-Seq has also been developed based on sorting p24 + cells from peripheral blood mononuclear cells of people living with HIV into individual wells by fluorescence-activated cell sorting [58], followed by single-cell whole genome amplification resulting in near full-length proviral sequencing, integration site analysis, and TCR sequencing. These approaches, if applied to CSF cells or preserved brain tissue, would provide valuable insights into CNS compartmentalization and reservoir at single cell level.

\section{Long-read Single-cell RNA Sequencing to Characterize HIV-1 Isoforms}

The most commonly used methods for single-cell RNA sequencing, including the $10 \times$ Genomics platform, result in short reads, thus limiting the detection of fulllength HIV RNA transcripts and detailed characterization of HIV RNA splicing in single cells. Notably, a full-length mRNA analysis of single cells termed singlecell isoform RNA-Seq (ScISOr-Seq) has been developed and utilized to identify cell-type specific RNA isoforms in the brain [59]. The approach uses the $10 \times$ Genomics platform to to amplify full-length cDNA from single cells and splits the cDNA into two pools that will be used for short-read high-throughput sequencing and long-read sequencing for isoform identification. A limitation of the long-read sequencing platform is read throughput, which limits the identification of rare transcripts of interest such as viral transcripts. To overcome sequencing depth limitations of long-read sequencing and the rarity of HIV RNA cDNA among host immune cellular transcripts, future approaches will need to leverage enrichmentbased strategies and amplification to achieve adequate coverage of full-length HIV transcripts. Indeed, enrichment and amplification approaches have been developed for HIV that account for sequence diversity $[60,61]$. We envision this method would improve knowledge of HIV splicing, viral diversity and evolution, CNS compartamentalization, and HIV host chimeric RNAs.

\section{Multimodal Single-cell Methods}

Single-cell methods that capture parallel information beyond two modalities-including transcription, protein, DNA methylation, chromatin, mtDNA, and other molecular information - from the same cell are rapidly advancing from hundreds of cells to millions of cells [62-66]. Joint profiling of histone modifications and gene expression from single cells, in methods like Paired-Tag [67] and scATACSeq [51], permit the mapping of the chromatin accessibility landscape of the host genome and accessibility state of the viral genome at single-cell resolution. Newer methods allow mitochondrial DNA to be amplified as well [68]. These permit the ability to examine mitochondrial DNA mutation rates across single cells and use this information to infer cell lineages and clonal expansion. A method to jointly profile chromatin accessibility of host cells and Tn5 accessible viral integration sites at single-cell levels termed EpiVIA was recently demonstrated in a chimeric antigen receptor T immunotherapy study [69••]. The approach relies on the hyperactive Tn5 transposase being able to transpose both accessible host DNA and accessible integrated proviral DNA. To identify accessible viral integration sites, hostviral fragments are identified bioinformatically and aligned to the human genome to localize viral integration sites. Moreover, an added layer of information about accessible viral integration sites would also be included in the assays readout. A limitation of this assay would be that inaccessible provirus would not be captured; hence, capturing all viral integration sites at single-cell resolution would not be feasible. Due to limitations of read length, the viral characteristics of integrated virus would not be attained and prevent the assessment of intact replication-competent virus. This method could be complemented with methods that profile matched HIV integration site and proviral sequencing (MIP-Seq) [70]. 
Fig. 1 Conceptual single-cell multimodal study of HIV in the CNS using CSF in PLWH

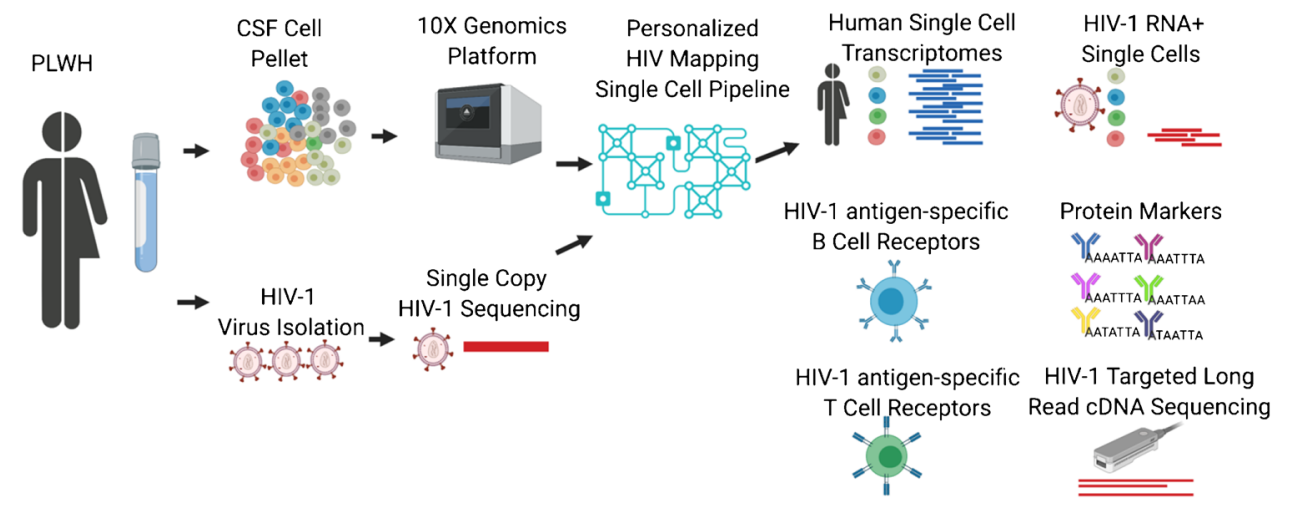

\section{Conclusions}

A major challenge of profiling CNS cells, including CSF cells and cells obtained from brain autopsy, is the need to assess fresh cells or cells that are stored to reliably preserve transcripts. Advances in CSF cell cryopreservation will advance single-cell studies of CSF to allow for assessment of biobanked samples [71]. Single nuclei sequencing and emerging spatial multiomics sequencing methods of fresh frozen postmortem brain tissues specimens likewise presents the important opportunity for single-cell studies of both the host and viral transcriptomes. Overall, tailoring single-cell sequencing methods to HIV permits the identification of disease-related cell subtypes, rare HIV-infected cells, HIV life cycle among single cells, HIV latency cell phenotypes, HIV reservoirs across compartments, interrogation of antigen specificity and immune system diversity, and therapeutic responses [72]. In the future, this approach can be guided by multidisciplinary teams that will use a personalized medicine approach to sequence the swarm of HIV diversity in a PLWH and use this data to more precisely identify HIVinfected cells and uncloak the molecular identities of latent cells that are barrier to cure (Fig. 1).

Fresh CSF cells would contain oligonucleotide-tagged antibodies defining surface proteins that would combine with the obtained transcriptomic data. Moreover, this single-cell dataset would also include the capability to examine HIV antigen-specific B cell and T cells with receptor sequences. Lastly, using a portion of cDNA for full-length DNA sequencing of enriched HIV sequences would permit interrogation of the virus. A single cell from CSF could contain 5 layers of unique information (host transcription, viral transcription, immune cell receptor sequence, surface protein levels, and full-length HIV transcript variation) that would provide unprecedented information about HIV RNA + containing cells in the CNS.

Author Contribution MC and SFF together wrote the manuscript.
Funding SFF is supported by K23MH118999, R01AI157488, and the Doris Duke Fund to Retain Clinician Scientists at Yale. MJC is supported by K01HL140271 and R01AG063846.

\section{Declarations}

Conflict of Interest The authors declare no competing interests.

\section{References}

Papers of particular interest, published recently, have been highlighted as:

- Of importance

$\bullet$ Of major importance

1. Chun T-W, Moir S, Fauci AS. HIV reservoirs as obstacles and opportunities for an HIV cure. Nat Immunol. 2015;16:584-9.

2. Ndung'u T, McCune JM, Deeks SG. Why and where an HIV cure is needed and how it might be achieved. Nature. 2019;576:397-405.

3. Hütter G, Nowak D, Mossner M, Ganepola S, Müssig A, Allers $\mathrm{K}$, et al. Long-term control of HIV by CCR5 Delta32/Delta32 stem-cell transplantation. N Engl J Med. 2009;360:692-8.

4. Gupta RK, Abdul-Jawad S, McCoy LE, Mok HP, Peppa D, Salgado $\mathrm{M}$, et al. HIV-1 remission following CCR5 $\Delta 32 / \Delta 32$ haematopoietic stem-cell transplantation. Nature. 2019;568:244-8.

5. Richman DD, Margolis DM, Delaney M, Greene WC, Hazuda D, Pomerantz RJ. The challenge of finding a cure for HIV infection. Science. 2009;323:1304-7.

6. Pitman MC, Lau JSY, McMahon JH, Lewin SR. Barriers and strategies to achieve a cure for HIV. Lancet HIV. 2018;5:e317-28.

7. Handoko R, Chan P, Jagodzinski L, Pinyakorn S, Ubolyam S, Phanuphak N, et al. Minimal detection of cerebrospinal fluid escape after initiation of antiretroviral therapy in acute HIV-1 infection. AIDS. 2021;35:777-82.

8. Valcour V, Chalermchai T, Sailasuta N, Marovich M, Lerdlum $\mathrm{S}$, Suttichom D, et al. Central nervous system viral invasion and inflammation during acute HIV infection. J Infect Dis. 2012;206:275-82.

9. Gelman BB, Lisinicchia JG, Morgello S, Masliah E, Commins D, Achim CL, et al. Neurovirological correlation with HIV-associated neurocognitive disorders and encephalitis 
in a HAART-era cohort. J Acquir Immune Defic Syndr. 2013;62:487-95.

10. Lamers SL, Rose R, Maidji E, Agsalda-Garcia M, Nolan DJ, Fogel GB, et al. HIV DNA is frequently present within pathologic tissues evaluated at autopsy from combined antiretroviral therapy-treated patients with undetectable viral loads. J Virol. 2016;90:8968-83.

11. Schnell G, Joseph S, Spudich S, Price RW, Swanstrom R. HIV-1 replication in the central nervous system occurs in two distinct cell types. PLoS Pathog. 2011;7:e1002286.

12. Yilmaz A, Price RW, Spudich S, Fuchs D, Hagberg L, Gisslén M. Persistent intrathecal immune activation in HIV-1-infected individuals on antiretroviral therapy. J Acquir Immune Defic Syndr. 2008;47:168-73.

13. Abdulle S, Hagberg L, Svennerholm B, Fuchs D, Gisslén M. Continuing intrathecal immunoactivation despite two years of effective antiretroviral therapy against HIV-1 infection. AIDS. 2002;16:2145-9.

14. JessenKrut J, Mellberg T, Price RW, Hagberg L, Fuchs D, Rosengren L, et al. Biomarker evidence of axonal injury in neuroasymptomatic HIV-1 patients. PLoS One. 2014;9:e88591.

15. Staprans S, Marlowe N, Glidden D, Novakovic-Agopian T, Grant RM, Heyes M, et al. Time course of cerebrospinal fluid responses to antiretroviral therapy: evidence for variable compartmentalization of infection. AIDS. 1999;13:1051-61.

16. Chan P, Hellmuth J, Colby D, Kroon E, Sacdalan C, Fletcher J, et al. Safety of lumbar puncture procedure in an international research setting during acute HIV infection. J Virus Erad. 2018;4:16-20. This report confirms safety and feasibility of lumbar puncture in international research setting.

17. Zhu C, Preissl S, Ren B. Single-cell multimodal omics: the power of many. Nat Methods. 2020;17:11-4.

18. Zanini F, Pu S-Y, Bekerman E, Einav S, Quake SR. Single-cell transcriptional dynamics of flavivirus infection. Elife [Internet]. 2018;7. Available from: https://doi.org/10.7554/eLife.32942

19. Heming M, Li X, Räuber S, Mausberg AK, Börsch A-L, Hartlehnert M, et al. Neurological manifestations of COVID-19 feature $\mathrm{T}$ cell exhaustion and dedifferentiated monocytes in cerebrospinal fluid. Immunity. 2021;54:164-175.e6.

20. Song E, Bartley CM, Chow RD, Ngo TT, Jiang R, Zamecnik $\mathrm{CR}$, et al. Exploratory neuroimmune profiling identifies CNSspecific alterations in COVID-19 patients with neurological involvement [Internet]. bioRxiv. 2020 [cited 2021 Mar 31]. p. 2020.09.11.293464. Available from: https://doi.org/10.1101/ 2020.09.11.293464v2

21. Onorati M, Li Z, Liu F, Sousa AMM, Nakagawa N, Li M, et al. Zika virus disrupts phospho-TBK1 localization and mitosis in human neuroepithelial stem cells and radial glia. Cell Rep. 2016;16:2576-92.

22. •• Gantner P, Pagliuzza A, Pardons M, Ramgopal M, Routy J-P, Fromentin R, et al. Single-cell TCR sequencing reveals phenotypically diverse clonally expanded cells harboring inducible HIV proviruses during ART. Nat Commun. 2020;11:4089. This study uses single-cell TCR sequencing and finds that antigendriven clonal expansion of $\mathbf{T}$ cells is a major contributor to viral persistence.

23. Wang S, Zhang Q, Hui H, Agrawal K, Karris MAY, Rana TM. An atlas of immune cell exhaustion in HIV-infected individuals revealed by single-cell transcriptomics. Emerg Microbes Infect. 2020;9:2333-47.

24.• Liu R, Yeh Y-HJ, Varabyou A, Collora JA, Sherrill-Mix S, Talbot CC Jr, et al. Single-cell transcriptional landscapes reveal HIV-1-driven aberrant host gene transcription as a potential therapeutic target. Sci Transl Med [Internet]. 2020;12. Available from: https://doi.org/10.1126/scitranslm ed.aaz0802. This study introduces SortSeq to isolate rare,
HIV-infected cells and perform transcriptomics on the isolated cells.

25. $\bullet$ Kazer SW, Aicher TP, Muema DM, Carroll SL, OrdovasMontanes J, Miao VN, et al. Integrated single-cell analysis of multicellular immune dynamics during hyperacute HIV-1 infection. Nat Med. 2020;26:511-8. This study performs scRNAseq on peripheral blood from participants longitudinally who became infected with HIV and describes cellular immune changes.

26. Cohn LB, da Silva IT, Valieris R, Huang AS, Lorenzi JCC, Cohen YZ, et al. Clonal CD4+ T cells in the HIV-1 latent reservoir display a distinct gene profile upon reactivation. Nat Med. 2018;24:604-9.

27. Tang F, Barbacioru C, Wang Y, Nordman E, Lee C, Xu N, et al. mRNA-Seq whole-transcriptome analysis of a single cell. Nat Methods. 2009;6:377-82.

28. Wang Y, Navin NE. Advances and applications of single-cell sequencing technologies. Mol Cell. 2015;58:598-609.

29. Papalexi E, Satija R. Single-cell RNA sequencing to explore immune cell heterogeneity. Nat Rev Immunol. 2018;18:35-45.

30. Macosko EZ, Basu A, Satija R, Nemesh J, Shekhar K, Goldman $M$, et al. Highly parallel genome-wide Expression profiling of individual cells using nanoliter droplets. Cell. 2015;161:1202-14.

31. Klein AM, Mazutis L, Akartuna I, Tallapragada N, Veres A, Li $\mathrm{V}$, et al. Droplet barcoding for single-cell transcriptomics applied to embryonic stem cells. Cell. 2015;161:1187-201.

32. Zheng GXY, Terry JM, Belgrader P, Ryvkin P, Bent ZW, Wilson $\mathrm{R}$, et al. Massively parallel digital transcriptional profiling of single cells. Nat Commun. 2017;8:14049.

33. Stoeckius M, Zheng S, Houck-Loomis B, Hao S, Yeung BZ, Mauck WM 3rd, et al. Cell Hashing with barcoded antibodies enables multiplexing and doublet detection for single cell genomics. Genome Biol. 2018;19:224.

34. - McGinnis CS, Patterson DM, Winkler J, Conrad DN, Hein MY, Srivastava V, et al. MULTI-seq: sample multiplexing for single-cell RNA sequencing using lipid-tagged indices. Nat Methods. 2019;16:619-26. This study introduces a lipidtagging method for cells enabling high-throughput sample indexing.

35. Baxter AE, O'Doherty U, Kaufmann DE. Beyond the replication-competent HIV reservoir: transcription and translationcompetent reservoirs. Retrovirology. 2018;15:18.

36. Sano T, Smith CL, Cantor CR. Immuno-PCR: very sensitive antigen detection by means of specific antibody-DNA conjugates. Science. 1992;258:120-2.

37. Stoeckius M, Hafemeister C, Stephenson W, Houck-Loomis B, Chattopadhyay PK, Swerdlow H, et al. Simultaneous epitope and transcriptome measurement in single cells. Nat Methods. 2017;14:865-8 undefined. Using the technology decsribed here, single cells can be simultaneously characterized by their transcriptome and by expression of cell surface proteins

38. Peterson VM, Zhang KX, Kumar N, Wong J, Li L, Wilson DC, et al. Multiplexed quantification of proteins and transcripts in single cells. Nat Biotechnol. 2017;35:936-9.

39. Shahi P, Kim SC, Haliburton JR, Gartner ZJ, Abate AR. Abseq: Ultrahigh-throughput single cell protein profiling with droplet microfluidic barcoding. Sci Rep. 2017;7:44447.

40. Chung H, Parkhurst CN, Magee EM, Phillips D, Habibi E, Chen $\mathrm{F}$, et al. Simultaneous single cell measurements of intranuclear proteins and gene expression [Internet]. bioRxiv. 2021 [cited 2021 Mar 31]. p. 2021.01.18.427139. Available from: https:// doi.org/10.1101/2021.01.18.427139v1.full

41. Pardons M, Baxter AE, Massanella M, Pagliuzza A, Fromentin R, Dufour C, et al. Single-cell characterization and quantification 
of translation-competent viral reservoirs in treated and untreated HIV infection. PLoS Pathog. 2019;15:e1007619.

42. • Ramesh A, Schubert RD, Greenfield AL, Dandekar R, Loudermilk R, Sabatino JJ Jr, et al. A pathogenic and clonally expanded B cell transcriptome in active multiple sclerosis. Proc Natl Acad Sci U S A. 2020;117:22932-43. By combining single-cell transcriptome and combined $B$ cell receptor sequencing, the authors find $B$ cell biological pathways that are more pronounced in in the spinal fluid of patients with MS, demonstrating feasibility and application of single BCR sequencing in neuroinflammatyory disease.

43. Esaulova E, Cantoni C, Shchukina I, Zaitsev K, Bucelli RC, Wu GF, et al. Single-cell RNA-seq analysis of human CSF microglia and myeloid cells in neuroinflammation. Neurol Neuroimmunol Neuroinflamm [Internet]. 2020;7. Available from: https://doi. org/10.1212/NXI.0000000000000732

44.•• Gate D, Saligrama N, Leventhal O, Yang AC, Unger MS, Middeldorp J, et al. Clonally expanded CD8 T cells patrol the cerebrospinal fluid in Alzheimer's disease. Nature. 2020;577:399404. Using single-cell TCR seqeuncing of CSF cells the authors find expansion of Epstein-Barr virus reactive $T$ cells in the CSF of people with Alzheimer's disease, and suggest an association between expanded cytotoxic $\mathrm{CD8} T$ cells clones and neurodegenerative disease.

45. Schafflick D, Xu CA, Hartlehnert M, Cole M, Schulte-Mecklenbeck A, Lautwein T, et al. Integrated single cell analysis of blood and cerebrospinal fluid leukocytes in multiple sclerosis. Nat Commun. 2020;11:247.

46. Farhadian SF, Mehta SS, Zografou C, Robertson K, Price RW, Pappalardo J, et al. Single-cell RNA sequencing reveals microglia-like cells in cerebrospinal fluid during virologically suppressed HIV. JCI Insight [Internet]. 2018;3. Available from: https://doi.org/10.1172/jci.insight.121718. This study describes the CSF immune cell landscape in PLWH and identifies a CSF-only population of microglia-like cells.

47. Pappalardo JL, Zhang L, Pecsok MK, Perlman K, Zografou C, Raddassi K, et al. Transcriptomic and clonal characterization of $\mathrm{T}$ cells in the human central nervous system. Sci Immunol [Internet]. 2020;5. Available from: https://doi.org/10.1126/sciim munol.abb8786

48. Kessing CF, Spudich S, Valcour V, Cartwright P, Chalermchai T, Fletcher JLK, et al. High number of activated CD8+ T cells targeting HIV antigens are present in cerebrospinal fluid in acute HIV infection. J Acquir Immune Defic Syndr. 2017;75:108-17.

49. Overall SA, Toor JS, Hao S, Yarmarkovich M, O'Rourke Sara M, Morozov GI, et al. High throughput pMHC-I tetramer library production using chaperone-mediated peptide exchange. Nat Commun. 2020;11:1909.

50. Setliff I, Shiakolas AR, Pilewski KA, Murji AA, Mapengo RE, Janowska K, et al. High-throughput mapping of B cell receptor sequences to antigen specificity. Cell 2019;179:1636-1646. e15. This study demonstrates a high-throughput mapping of BCR sequence at single-cell levels with antigen specificity to identify HIV-specific B cells in PLWH.

51. Shema E, Bernstein BE, Buenrostro JD. Single-cell and single-molecule epigenomics to uncover genome regulation at unprecedented resolution. Nat Genet. Nature Publishing Group; $2018 ; 1$

52. Kaya-Okur HS, Wu SJ, Codomo CA, Pledger ES, Bryson TD, Henikoff JG, et al. CUT \& for efficient epigenomic profiling of small samples and single cells. Nat Commun. 2019;10:1930. This study developed a method to profile histone modifications and transcription factors at single-cell resolution.

53. Battivelli E, Dahabieh MS, Abdel-Mohsen M, Svensson JP, Tojal Da Silva I, Cohn LB, et al. Distinct chromatin functional states correlate with HIV latency reactivation in infected primary
CD4+ T cells. Elife [Internet]. 2018;7. Available from: https:// doi.org/10.7554/eLife.34655

54. Abdel-Mohsen M, Richman D, Siliciano RF, Nussenzweig MC, Howell BJ, Martinez-Picado J, et al. Recommendations for measuring HIV reservoir size in cure-directed clinical trials. Nat Med. 2020;26:1339-50.

55. Chaillon A, Gianella S, Dellicour S, Rawlings SA, Schlub TE, De Oliveira MF, et al. HIV persists throughout deep tissues with repopulation from multiple anatomical sources. J Clin Invest. 2020;130:1699-712.

56. Plaza-Jennings A. Cell-type specific genomics and transcriptomics of HIV in the braiN. March 6-10, 2021.

57. Sun C, Liu L, Pérez L, Li X, Liu Y, Xu P, et al. Droplet microfluidic sequencing of HIV genomes and integration sites [Internet]. bioRxiv. 2020 [cited 2021 Mar 31]. p. 2020.09.25.314120. Available from: https://doi.org/10.1101/2020.09.25.314120v1. full

58. Cole B, Lambrechts L, Gantner P, Noppe Y, Bonine N, Witkowski W, et al. In-depth single-cell analysis of translationcompetent HIV-1 reservoirs identifies cellular sources of plasma viremia [Internet]. bioRxiv. 2021 [cited 2021 Mar 31]. p. 2021.02.15.431218. Available from: https://doi.org/10.1101/ 2021.02.15.431218v1

59. Gupta I, Collier PG, Haase B, Mahfouz A, Joglekar A, Floyd $\mathrm{T}$, et al. Single-cell isoform RNA sequencing characterizes isoforms in thousands of cerebellar cells. Nat Biotechnol [Internet]. 2018; Available from: https://doi.org/10.1038/nbt.4259

60. Iwase SC, Miyazato P, Katsuya H, Islam S, Yang BTJ, Ito J, et al. HIV-1 DNA-capture-seq is a useful tool for the comprehensive characterization of HIV-1 provirus. Sci Rep. 2019;9:12326.

61. Yamaguchi J, Olivo A, Laeyendecker O, Forberg K, Ndembi N, Mbanya D, et al. Universal target capture of HIV sequences from NGS libraries. Front Microbiol. 2018;9:2150.

62. Cao J, Cusanovich DA, Ramani V, Aghamirzaie D, Pliner HA, Hill AJ, et al. Joint profiling of chromatin accessibility and gene expression in thousands of single cells. Science. 2018;361:1380-5.

63. Chen S, Lake BB, Zhang K. High-throughput sequencing of the transcriptome and chromatin accessibility in the same cell. Nat Biotechnol. 2019;37:1452-7.

64. Clark SJ, Argelaguet R, Kapourani C-A, Stubbs TM, Lee HJ, Alda-Catalinas C, et al. scNMT-seq enables joint profiling of chromatin accessibility DNA methylation and transcription in single cells. Nat Commun. 2018;9:781.

65. Zhu C, Yu M, Huang H, Juric I, Abnousi A, Hu R, et al. An ultra high-throughput method for single-cell joint analysis of open chromatin and transcriptome. Nat Struct Mol Biol. 2019;26:1063-70.

66. Xing QR, Farran CAE, Zeng YY, Yi Y, Warrier T, Gautam P, et al. Parallel bimodal single-cell sequencing of transcriptome and chromatin accessibility. Genome Res. 2020;30:1027-39.

67. Zhu C, Zhang Y, Li YE, Lucero J, Behrens MM, Ren B. Joint profiling of histone modifications and transcriptome in single cells from mouse brain. Nat Methods. 2021;18:283-92.

68. Xu J, Nuno K, Litzenburger UM, Qi Y, Corces MR, Majeti $\mathrm{R}$, et al. Single-cell lineage tracing by endogenous mutations enriched in transposase accessible mitochondrial DNA. Elife [Internet]. 2019;8. Available from: https://doi.org/10.7554/eLife. 45105

69.•• Wang W, Fasolino M, Cattau B, Goldman N, Kong W, Frederick MA, et al. Joint profiling of chromatin accessibility and CAR-T integration site analysis at population and single-cell levels. ProcNatl Acad Sci U S A [Internet]. National Academy of Sciences; 2020 [cited $2020 \mathrm{Feb} 25$ ]; Available from: https://www. pnas.org/content/early/2020/02/19/1919259117. This work demonstrates the use of single-cell ATAC sequencing data 
for jointly profiling chromatin accessiblity and Tn5 accessible viral integration sites at single-cell level.

70. Einkauf KB, Lee GQ, Gao C, Sharaf R, Sun X, Hua S, et al. Intact HIV-1 proviruses accumulate at distinct chromosomal positions during prolonged antiretroviral therapy. J Clin Invest [Internet]. 2019; Available from: https://doi.org/10.1172/JCI12 4291

71. Oh H, Leventhal O, Channappa D, Henderson VW, Wyss-Coray $\mathrm{T}$, Lehallier B, et al. Methods to investigate intrathecal adaptive immunity in neurodegeneration. Mol Neurodegener. 2021;16:3.
72. Sannier G, Dubé M, Kaufmann DE. Single-cell technologies applied to HIV-1 research: reaching maturity. Front Microbiol. 2020;11:297.

Publisher's Note Springer Nature remains neutral with regard to jurisdictional claims in published maps and institutional affiliations. 